\title{
El Acceso y la Retención en la Educación Superior de Estudiantes con Discapacidad en Ecuador
}

\author{
Carlos X. Espinosa ${ }^{(1)}$, Víctor G. Gómez ${ }^{(1)}$ y Carlos M. Cañedo ${ }^{(2)}$ \\ (1) Universidad Metropolitana, Ciudadela Garzota primera etapa, Mz 23, Solares 7 y 8 , \\ Av.Miguel H. Jijón y Av. Guillermo Pareja Rolando, Guayaquil-Ecuador \\ (e-mail: cespinoza@umet.edu.ec; vgomez@umet.edu.ec) \\ (2) Universidad de Cienfuegos, Carretera a Rodas Km. 4 Cuatro Caminos, Cienfuegos-Cuba \\ (e-mail: ccanedo@ucf.edu.cu)
}

Recibido May. 24, 2012; Aceptado Jun. 21, 2012; Versión final recibida Ago. 20, 2012

\section{Resumen}

El objetivo del trabajo presentado es la propuesta de un modelo para la gestión del proceso de acceso y retención de estudiantes universitarios con discapacidad desde una concepción pedagógica. Se estudió el comportamiento de la problemática a nivel regional a partir de una muestra de universidades de la provincia del Guayas. Se propuso el Programa de Carrera Docente dirigido a la profesionalización del claustro universitario como vía para la implementación y soporte a las etapas que conforman el modelo. Finalmente se concluye que, a pesar de ser política de estado, las estrategias desarrolladas para el acceso a la educación superior de estudiantes discapacitados no se materializan en acciones concretas y medibles. Se privilegia la oferta de un tratamiento especial en el orden financiero para estos estudiantes.

Palabras clave: discapacidad, acceso y retención, educación superior, necesidades educativas

\section{Access and Retention in Higher Education of Students with Disabilities in Ecuador}

\begin{abstract}
This work presents a model for managing the process of access and retention of university students with disabilities in Ecuador based on a pedagogical conception. The behavior of this problem was studied in some universities of the province of Guayas. The proposed Teaching Career Program is aimed at the professionalization of university teaching as a way to implement the remaining steps of the model. It is concluded that despite being a government policy, the strategies developed for disabled students do not materialize into concrete and measurable actions. The system mainly focuses on providing especial financial treatment to these students.
\end{abstract}

Keywords: disabilities, access and retention, higher education, educational needs 


\section{INTRODUCCIÓN}

La calidad educativo - formativa de los procesos de enseñanza y aprendizaje está estrechamente vinculada a los niveles de éxito logrados al finalizar los períodos lectivos y, con ello, a índices de repetición, asistencia, abandono, logros en evaluaciones estandarizadas, etc. Dichos indicadores a partir de los estudios sobre la realidad educativa del entorno permiten emitir juicios sobre la calidad de la formación, la profesionalización del claustro universitario y los niveles de excelencia de su práctica pedagógica, la eficiencia y eficacia del proceso de enseñanza y aprendizaje así como la implicación organizacional en el establecimiento y control de estrategias de desarrollo de capacidades en los actores implicados en el proceso.

La literatura científica da cuenta de la existencia de una amplia gama de definiciones alrededor de la preservación y garantías de derecho de los sectores vulnerables a la marginación social, a saber: integración, igualdad de oportunidades, inclusión, etc. La noción de igualdad es parte de la idea de ciudadanía y no habrá integración auténtica en tanto las diferencias no sean preservadas como garante de la integración (Kovadloff, 2003). Independientemente de ello, la realidad cotidiana y sus disímiles escenarios conllevan a afirmar que, dentro del espectro de sectores sociales marginados, la discapacidad constituye en sí misma una variable que condiciona significativamente la ubicación de los individuos dentro del entramado social pues determina las posiciones educativo - formativas, económicas, políticas y normativas para quienes la padecen y también, de manera estructuralmente diferente, para quienes no. Las personas con discapacidad conforman la minoría del mundo más grande y en mayor desventaja. Se estima que el 20 por ciento de las personas más pobres del mundo son personas con discapacidad; que el 98 por ciento de los niños con discapacidad en los países en desarrollo no asisten a la escuela; que un 30 por ciento de los niños de la calle en el mundo son niños con discapacidad y que sólo el 3 por ciento de los adultos con discapacidad saben leer y escribir. "(...) las personas con discapacidad (...) tienen poca esperanza de ir a la escuela, conseguir un trabajo, (...) disfrutar de una vida social o votar (...) las tiendas, los servicios y transportes públicos y hasta la información, están fuera de su alcance" (ONU, 2007:1)

En el tema de inclusión educativa, se toma como fundamento el concepto de diversidad manejado en la literatura científica (García, 1995, 2005; Jiménez y Vilá, 1999; Arnaiz, 2003; Sánchez y Torres, 2004; Sánchez y Pulido, 2007; Sánchez, 2011), partiéndose del hecho de que los servicios educativos deben estar pensados para todos y que cualquier alumno puede participar de ellos en igualdad de condiciones. En ese sentido, los Ministros de Educación reunidos en mayo de 2008 en El Salvador llegan al consenso de que la educación es la herramienta fundamental para el desarrollo de Iberoamérica, acordaron emprender acciones que posibilitaran concretar la propuesta "Metas Educativas 2021: la educación que queremos para la generación de los Bicentenarios". El objetivo consiste en lograr que más estudiantes ingresen al sistema y permanezcan en él durante más tiempo, con una oferta de calidad reconocida e inclusiva. De las once metas, la segunda se refiere a la educación en la diversidad y expresa que: "La diversidad de alumnos y de alumnas es la situación habitual en las escuelas y en la sociedad. La meta que se plantea pretende que los sistemas educativos fortalezcan las políticas atentas a la diversidad y compensadoras de sus insuficiencias de partida, de manera especial en la defensa de los derechos de las alumnas y en el apoyo a la inclusión social y educativa de los alumnos más vulnerables, especialmente las minorías étnicas, las poblaciones indígenas y afrodescendientes, los que se encuentran en condiciones sociales desfavorables y los alumnos que presentan necesidades educativas especiales asociadas a condiciones de discapacidad" (OEI 2008: 99-101).

La UNESCO ha identificado cuatro elementos clave que como definitorios en las prácticas de inclusión de los grupos en desventaja. El primero de ellos reconoce la inclusión como un proceso y defiende la posición de que se debe contemplar la inclusión como una búsqueda interminable de mejores formas de respuesta a la diversidad. El segundo elemento apunta al hecho de que la inclusión supone la identificación y retirada de barreras y, en consecuencia, implica recopilar, relacionar y evaluar información para planificar mejoras en las políticas y en la práctica. El tercer elemento establece que la inclusión tiene relación con la presencia, participación y éxito escolar de todos los alumnos mientras que el cuarto deja claro que la inclusión implica un énfasis especial 
en aquellos grupos de alumnos que pueden estar en riesgo de marginación, exclusión o fracaso escolar y supone una responsabilidad moral de garantizar que se tomen las medidas necesarias para garantizar la presencia, participación y éxito escolar en el sistema educativo de dichos grupos (UNESCO, 2003)

En el caso del acercamiento de la universidad a la inclusión social y, en específico, a la aceptación de las diferencias, características y necesidades de las personas con discapacidad el tema se vuelve controvertido y su tratamiento tiene matices de superficialidad extrema aun cuando el discurso parte del reconocimiento de que la diversidad social constituye un componente fundamental que merece ser revalorizado desde los puntos de vista académico y de gestión en las instituciones de educación superior. La Universidad inclusiva debe ser aquel lugar donde todos son aceptados y apoyados por todos para que tengan cubiertas sus necesidades educativas, la idea debe ser replanteada de manera que se interiorice loa necesidad del tránsito de una universidad basada en la evaluación de la competencia docente o la asimilación de las competencias generales y específicas en los estudiantes a una universidad donde se evalúe adicionalmente la cooperación entre pares para favorecer la inclusión (estudiante - estudiante, docente - docente, docente - estudiante, etc.). De ello dependerá que el paso del estudiante con discapacidad por las aulas universitarias se convierta en un mecanismo importante de integración social o sea percibido, por él mismo y el resto de los actores universitarios, como una forma más de exclusión que degenerará en deserción del medio universitario. A propósito de ello, Puigdellívol (2007) explica estas relaciones como un punto importante en el deseo de aprender de los estudiantes y como una respuesta a sus necesidades en el caso de que la institución educativa asuma los retos de la inclusión.

La sociedad actual, como sociedad de derecho, ha crecido en lo referente a la promulgación de leyes y normativas a favor de la inclusión y con la clara intencionalidad de proteger a las personas con discapacidad como uno de los sectores tradicionalmente excluidos y más desprotegidos, por su masiva representatividad y por la gran cantidad de recursos que se necesitan para garantizar plenos accesos a determinados sectores como el educativo. Aun así, todas estas leyes y regulaciones gubernamentales han contribuido a mejorar el acceso a las matrículas de los estudiantes con discapacidad en las Instituciones de Educación Superior (IES). Paul (2000:209) sugiere que "estos estudiantes se enfrentan constantemente a varias barreras en su ambiente educativo". Sorprendentemente, existen pocas investigaciones que profundizan en la naturaleza de estas barreras (Baron et al., 1996). En la década pasada aparecieron algunos estudios que proponían guías y daban recomendaciones de buenas prácticas con orientaciones para hacer posible la inclusión de estudiantes con discapacidad al entorno universitario (Doyle y Robson, 2002; Gravestock y Healey, 2001; Teachability, 2000) pocas han sido sistematizadas en la actualidad. No hace más de una década que las investigaciones dirigidas a la inclusión de estudiantes con discapacidad en los sistemas educativos comienzan a tocar el tema de la inclusión en las aulas universitarias y se han concentrado en lo principal en el estudio de las actitudes, valoraciones, percepciones, etc. profundizando poco en el tema de las aptitudes de los diferentes actores universitarios para enfrentar esta problemática educativa (Freewood y Spriggs, 2003; Fuller et al., 2004a; Fuller et al., 2004b).

Constituye una realidad el hecho de que muchos modelos de evaluación externa a instituciones de educación superior en el mundo y en especial en Ecuador, sobre todo los aplicados por el desaparecido Consejo Nacional de Evaluación y Acreditación (CONEA) y el actual Consejo de Evaluación, Acreditación y Aseguramiento de la Calidad de la Educación Superior (CEAACES), se dedican más a la valoración del acceso y la inclusión en general desde términos obsoletos y/o minimalistas que constituyen obligaciones establecidas por las normativas vigentes como lo son los acondicionamientos arquitectónicos y la eliminación de barreras de acceso al medio físico (rampas, elevadores) así como otros indicadores relacionados con la cantidad de becas y los criterios de asignación penetrando menos en temas más sensibles como lo son las adecuaciones curriculares, las garantías del pleno acceso a la información de los programas y materias, los ambientes de aprendizaje, las formas y diferenciación en la evaluación, los acompañamientos, la preparación pedagógica del claustro para asumir el reto de la inclusión en el aula universitaria, etc. Como Muñoz y Maruny (1993) señalan "El principal problema para desarrollar una pedagogía de 
la diversidad no son tanto los instrumentos didácticos necesarios, como las convicciones sociales, culturales y pedagógicas del profesorado, alumnado y los mismos padres y madres". En este sentido, Aponte (2008) deja claro que dichos "procesos deberían estar acompañados por la generación de condiciones de aprendizaje que tengan en cuenta las necesidades de los grupos tradicionalmente excluidos y sus niveles de rezago en capacidad cognitiva y conocimiento para operar efectivamente en la reducción de la desigualdad y el aumento de la cohesión social" (Aponte, 2008).

Chiroleu (2011) considera que, en la educación superior latinoamericana se mantienen "procesos de doble cara que dan cuenta de transformaciones inacabadas o inconclusas que tienden a potenciar las marcas de nacimiento (...)". Dichos procesos, al invocar como meta la modernización de los sistemas "(...) no hacen más que acentuar los rasgos más regresivos (...)". Más adelante acota que uno de los problemas de mayor alcance en la universidad latinoamericana lo constituye "la expansión de la cobertura sin reducción de las desigualdades sociales ni inclusión plena en la educación superior (...)". En otro de sus aportes, Chiroleu (2009) deja claro que, en el caso de la educación superior: "la relación inclusión - exclusión, adquiere connotaciones y alcances particulares "(...)" El debate actual en cambio, introduce nuevos elementos en la medida en que se apunta a incluir dentro de la universidad una diversidad racial, cultural y sexual semejante a la que existe en el seno de la sociedad, en procurar de remediar discriminaciones históricas que han conducido a la situación de desigualdad por la que atraviesan esos grupos. En este sentido, la implementación en algunos países de Políticas de Acción Afirmativa en el ámbito de la educación superior intenta lograr una mayor integración social en sociedades caracterizadas por fragmentaciones de diferente naturaleza".

Maingon en su artículo sobre la caracterización de los estudiantes con discapacidad en la Universidad Central de Venezuela resume algunos de los aspectos más relevantes sobre la inclusión de las personas con discapacidad en la educación superior al destacar que existen restricciones de importancia que dificultan el acceso, la permanencia y el egreso de las personas con discapacidad en el sistema de educación superior, que las experiencias que en materia de integración a la educación superior se reportan son aisladas y responden generalmente a iniciativas individuales o personalizadas y se refiere a la necesidad de las adaptaciones curriculares en todos los niveles de educación insistiendo en que, en el caso de la educación superior, se reporta lentitud y resistencia para la ejecución de las mismas (Maingon, 2007).

Otros autores como Alonso y Díez (2008) promueven el concepto de Diseño Universal para el Aprendizaje (DUA) basado en los avances sobre el aprendizaje y las nuevas tecnologías para responder a las diferencias individuales en los estudiantes descrito por Rose y Meyer (2000) en el artículo Universal Design for Learning cuya premisa fundamental se basa en considerar las necesidades del mayor número posible y todos los tipo de usuarios que puedan tenerse en cuenta para planificar y diseñar servicios, productos, instalaciones, etc. desde la etapa de diseño de los mismos de manera que se introduzcan las consideraciones que garanticen iguales oportunidades de acceso para todos. La propuesta del DUA no es otra que introducir en el curriculum, partiendo de su propia concepción, estrategias y métodos flexibles que posibiliten a los docentes estar un paso por delante cuando se tenga un alumno con necesidades específicas para el aprendizaje en el aula universitaria.

Es por ello que, las acciones que se desarrollen, con el fin de garantizar la calidad de la formación en los diferentes niveles, deben centrarse en la propuesta de un sistema articulado de investigación - acción, capacitación, acompañamiento, seguimiento y control del proceso de enseñanza y aprendizaje con énfasis en el desarrollo de competencias en el plano de la transformación de las prácticas pedagógicas de los docentes, dándoles la oportunidad de generar nuevas estrategias. El desarrollo de dichas competencias supone ampliar su capacidad de comprensión de los problemas de la práctica pedagógica y los desafíos que confrontan, en un contexto no de mejoramiento, sino de cambio en el desempeño. 


\section{DISCAPACIDAD Y EDUCACIÓN SUPERIOR. EL CASO ECUATORIANO}

La educación superior contemporánea ha tenido importantes cambios relacionados con los impactos que sufre en su relación con el entorno económico, los procesos de mercantilización de productos, los avances crecientes en las tecnologías y las repercusiones sociales de ellos en todos los ámbitos de la sociedad. Los más significativos se generan por la naturaleza contradictoria que esos procesos tienen para los países de menor desarrollo, donde sus consecuencias se amplifican.

Estos elementos han determinado el planteamiento, por parte de la UNESCO, de un conjunto de estrategias, orientadas a atenuar los impactos y superar las dificultades que se presentan en el sistema de educación superior. De estos esfuerzos dan cuenta, múltiples documentos políticos y de análisis especializados sobre el tema, desde fines de la década del 90 de siglo anterior y hasta la actualidad (UNESCO, 1996; Tunnerman, 1996; UNESCO, 1998; IESAL - UNESCO, 2006; García, 2008; UNESCO-IESALC, 2009). El documento "Superar la exclusión" (UNESCO, 2003) insiste en la necesidad de generar sistemas educativos integradores. El Marco de Acción sobre las Necesidades Educativas Especiales (UNESCO, 1994) menciona que son los organismos los que han de buscar la manera de garantizar el acceso para todos, o lo que es lo mismo, "... es la enseñanza la que debe adaptarse a las necesidades del estudiante, y no el estudiante a la norma". Para lograr el objetivo de la inclusión educativa en cualquier nivel, incluido el superior se debe luchar contra las barreras construidas por la tradición escolar, y reforzadas por determinadas culturas escolares, que tienden a limitar la presencia y las posibilidades de aprendizaje y participación de los estudiantes con discapacidad en condiciones de igualdad (Echeita, 2011).

Lograr la excelencia a partir de los estándares establecidos internacionalmente garantizando la pertinencia y hacerlo con equidad, parece ser un sueño de la universidad en el mundo y de la latinoamericana en particular. Sobre las contradicciones que se manifiestan en el medio académico para armonizar la misión social exigida a la educación superior, la literatura especializada señala que, al responder eficazmente a las demandas externas, la universidad actúa en forma pertinente solo cuando se plantea como objeto de investigación a su propio entorno (Brovetto, 1994).

Varios de los aspectos estratégicos que los cambios de la educación superior suponen, confluyen con el proceso de integración vertical y horizontal que, como regularidades de su desarrollo, se vienen manifestando en la ciencia y la tecnología. Desde esta perspectiva de análisis se muestra la imposibilidad de mantener los estancos cognoscitivos propios de la enseñanza superior. Por otra parte, se anuncia la necesaria atención a las necesidades sociales, ambientales, económicas y culturales que se generan en los contextos y escenarios donde se desarrolla la influencia de los centros de educación superior. El carácter complejo de los objetos, y las interacciones entre ellos generadas por dichas necesidades, reclama la interdisciplinariedad para su atención y la introducción de cambios importantes en el currículum universitario. Un ejemplo muy importante de ello lo constituyen las adaptaciones curriculares propias para el tratamiento a las personas con discapacidad.

Uno de los aspectos de mayor importancia, por sus implicaciones para los estudiantes con diferentes necesidades de aprendizaje, es su acceso, permanencia y progresión en el sistema educativo. Esta es una cuestión que repercute de manera decisiva en el desarrollo personal y la capacitación del estudiantado, condicionando sus oportunidades de formación profesional y, de hecho, de acceso al entorno laboral lo que le impide poder desarrollar una vida lo más independiente y normalizada posible (Alonso y Araoz, 2011).

En un estudio realizado por investigadores españoles, publicado en un número de la Revista de Educación dedicada a la formación práctica de estudiantes universitarios se demuestra que existe un alto grado de acuerdo entre los docentes en torno a que no se sienten con la suficiente competencia para dar una respuesta adecuada a los estudiantes con discapacidad lo que pone de manifiesto la necesidad de una formación pertinente como factor determinante ya que se reconoce escasa socialización de las experiencias de integración de estos estudiantes al aula universitaria y que la sistematización de las buenas prácticas es aún deficiente (Sánchez, A., 2011). 
En el caso de Ecuador, la Ley Orgánica de Educación Superior (LOES) declara el principio de pertinencia dentro de aquellos de los principios que rigen el sistema de Educación Superior en el país. El Artículo 107 dice textualmente que "(...) El principio de pertinencia y acceso consiste en que la educación superior responda a las expectativas y necesidades de la sociedad, a la planificación nacional, y al régimen de desarrollo, a la prospectiva de desarrollo científico, humanístico y tecnológico mundial, y a la diversidad cultural" (Ley Orgánica de Educación Superior, 2010 )

En Ecuador, como una de la vías para responder a las demandas del Programa nacional para el Buen Vivir y garantizar la pertinencia universitaria, respondiendo a las necesidades de la población y a la diversidad de un país rico en tradiciones y culturas, se articulan mecanismos que faciliten la igualdad de oportunidades, es decir, el acceso indistinto al proceso por el cual los diversos sistemas de la sociedad, el marco material y legal, los servicios, las actividades, la información y la documentación se dan de igual forma y de manera efectiva, accesible plenamente a todos y cada uno. Sin embargo, ¿cuántas universidades e instituciones superiores actúan en correspondencia con esta definición?, ¿cuántas universidades incluyen programas que favorecen el aprendizaje a estudiantes con necesidades diversas? El reclamo a la pertinencia se entiende en términos de que una institución debe ser capaz de conectarse al entorno en sus diferentes niveles (económico, académico, de gestión, social, cultural, tecnológico y científico) y escalas (local, nacional e internacional) sobre la base de los marcos regulatorios bajo los cuales funciona, atendiendo a las exigencias políticas y las trayectorias y capacidades académicas desarrolladas en la propia institución y que garanticen la igualdad de oportunidades para el acceso, la permanencia y el progreso en el medio universitario a todos y cada uno de los ciudadanos.

Ello constituye, sin dudas, una etapa de la madurez jurídica que al respecto ha venido ganando el sistema de derecho en el Ecuador. El 10 de agosto de 1992 se publica Ley sobre discapacidades y, posteriormente, en la nueva redacción de la constitución del país aprobada en el año 2008, queda reflejado en su sección sexta dedicada a personas con discapacidad, artículo 47 que "(...) el Estado garantizará políticas de prevención de las discapacidades y, de manera conjunta con la sociedad y la familia, procurará la equiparación de oportunidades para las personas con discapacidad y su integración social (Constitución de la República del Ecuador, 2008). Se reconoce a las personas con discapacidad, los derechos a: (...) Una educación que desarrolle sus potencialidades y habilidades para su integración y participación en igualdad de condiciones. Se garantizará su educación dentro de la educación regular. Los planteles regulares incorporarán trato diferenciado y los de atención especial la educación especializada. Los establecimientos educativos cumplirán normas de accesibilidad para personas con discapacidad e implementarán un sistema de becas que responda a las condiciones económicas de este grupo (...) La educación especializada para las personas con discapacidad intelectual y el fomento de sus capacidades mediante la creación de centros educativos y programas de enseñanza específicos (...) El acceso a mecanismos, medios y formas alternativas de comunicación, entre ellos el lenguaje de señas para personas sordas, el oralismo y el sistema braille" (Constitución de la República del Ecuador, 2008).

Resultado de este esfuerzo político y social es el estudio científico y médico sobre las discapacidades en el Ecuador conocido también como Misión Manuela Espejo, la que ha sido calificada como "(...) una cruzada sin precedentes en la historia del Ecuador (...) para determinar las causas de las discapacidades y conocer la realidad biopsicosocial de esta población desde los puntos de vista biológico, psicológico, social, clínico y genético" (Informe de la Misión Manuela Espejo, 2010:2). El estudio ha supuesto, por primera vez en la historia del país, la identificación, el diagnóstico y la articulación de múltiples proyectos encaminados a la mejora de las condiciones de vida de miles de personas con discapacidad en los más apartados y recónditos lugares del país.

En el año 2004 se publicaron los resultados del estudio sobre integración de las personas con discapacidad en la educación superior en Ecuador (Rodríguez, 2004) demostrándose que el acceso a la educación superior de éstas personas era insignificante estadísticamente. En él se señalaba que apenas el 1,8\% de la totalidad de la población discapacitada accedía a la educación superior y señalaba que persistían barreras que imposibilitan la integración en el nivel universitario 
a pesar de contarse con un marco legal integral, que en teoría, debía facilitar el acceso a todos los niveles, incluido el universitario. La realidad es muy distinta pues aún se evidencian restricciones de tipo económico, social, técnico y político que limitan profundamente el acceso a la educación superior de las personas con discapacidad.

Con pesar se debe reconocer que no se encontraron estudios más actualizados sobre el acceso a la educación universitaria ecuatoriana de las personas con discapacidad. Rodríguez (2004) afirma en su informe que en el Ecuador existen pocas bases de datos oficiales que registren las características de edad, sexo, procedencia, nivel de instrucción, empleo, condición socioeconómica, etc., ni se registra información oficial sobre los siguientes aspectos en las instituciones de educación superior: formación y capacitación, programas de sensibilización a la comunidad universitaria, limitación de barreras arquitectónicas, urbanísticas y de accesibilidad al transporte, adaptaciones tecnológicas y adaptaciones curriculares.

Surgen entonces algunas interrogantes sobre el papel que le corresponde protagonizar a la universidad ecuatoriana en la profesionalización del claustro universitario para lograr el acceso y retención de las personas con discapacidad. El cuestionamiento gira alrededor de las siguientes situaciones específicas: ¿cuáles son los criterios contemporáneos sobre el término de formación y discapacidad desde los diferentes modelos y enfoques curriculares?; ¿cuáles son las principales necesidades de profesores para llevar a vías de hecho el modelo que se elija?; ¿cómo articular el contenido teórico de las carreras y materias con las experiencias significativas del logro del acceso y retención de estudiantes con discapacidad desde el currículo universitario?; ¿cómo articularlas con proyectos que permitan vincular a los estudiantes con realidades sociales significativas específicamente con la sociedad ecuatoriana?; ¿cómo motivar a los actores del proceso a considerar su campo de formación profesional como objeto de estudio?; ¿cuáles son las dimensiones y componentes esenciales a tener en cuenta para la concepción de un modelo en el que participen todos los actores universitarios?

\section{FALENCIAS DE LA UNIVERSIDAD ECUATORIANA FRENTE A LA DISCAPACIDAD}

Teniendo en cuenta estos resultados se entiende que la sociedad ecuatoriana reclama a todos los niveles una contribución al mejoramiento de la calidad de vida de los ciudadanos con discapacidad, y al manejo responsable de estas situaciones. El propósito de la educación inclusiva es permitir que los profesores y estudiantes se sientan cómodos ante la diversidad y la perciban no como un problema, sino como un desafío y una oportunidad para enriquecer el entorno de aprendizaje. Basado en dicha convicción se realiza un estudio en la provincia del Guayas, Ecuador con el objetivo de diagnosticar los aspectos relacionados a la inserción, acceso, retención, tratamiento, flexibilidad y percepción de los diferentes actores en 11 Universidades de Guayaquil, incluida la Universidad Metropolitana.

Metodológicamente la investigación realizada se ubica en el paradigma naturalista ya que el análisis se efectúa en y desde el campo de acción y la realidad que se genera por el propio objeto de estudio, sin manipular ni alterar las circunstancias. Se utilizan entrevistas estructuradas dirigidas a los diferentes actores universitarios, directivos, docentes, responsables de la actividad académica, del departamento de bienestar universitario, estudiantes con discapacidad, e incluso, rectores de estas IES. Se trata de un diseño de tipo descriptivo y de modalidad selectiva. Se desestima la realización de un proceso de muestreo o de representatividad de la muestra trabajando solo con una muestra participada o aceptante productora de datos (Fox, 1981:38), a la que al aplicársele el test de validez muestral arroja un nivel de confianza del $95 \%$ y un margen de error inferior al $10 \%$.

Una vez elaborada la primera versión, el guión de la entrevista estructurada fue sometido a juicio de expertos y, con la intención de garantizar la calidad, capacidad para extraer la información necesaria, facilidad de comprensión por los entrevistados y reducir los posibles errores asociados a los instrumentos utilizados en la recogida de información se llevó a cabo un estudio piloto, aplicándose a diferentes actores de la Universidad Metropolitana con el objetivo de adecuarlas al objeto de estudio. Posteriormente, tras el análisis de los resultados, se modifica la redacción de 
algunas preguntas y se eliminan otras por no aportar información relevante obteniéndose las versiones definitivas de los guiones.

Las entrevistas realizadas tuvieron como objetivo global estudiar el nivel de inclusión educativa y social de los estudiantes con discapacidad, desde las perspectivas de cada uno de los actores mencionados con anterioridad. Se tenía la intención de identificar, en cada IES, lo relacionado con las estadísticas asociadas a la discapacidad (cantidad de estudiantes con discapacidad matriculados, cantidad de docentes con discapacidad contratados, cantidad de personal administrativo con discapacidad contratado en la IES y su caracterización en cuanto a elementos sociodemográficos como: tipo de discapacidad, carrera, sexo, edad, entre otros). Se buscaba también determinar las particularidades y tipos de adaptaciones curriculares aprobadas dirigidas a estudiantes con capacidad y necesidad diversa, la tecnología implementada y los materiales y medios didácticos existentes en la institución con el fin de favorecer la comunicación y el pleno acceso a la información en el proceso de enseñanza aprendizaje. Se profundizaba además, en la determinación de las estrategias desarrolladas en función del cambio de actitud pidiendo que se relacionaran las acciones de concienciación con docentes y estudiantes "sin discapacidad" y, por último, la existencia de "acomodos" especiales en la infraestructura y las condiciones de aprendizaje con el objetivo de dotar al estudiante, docente y empleado con discapacidad de un acceso adecuado al espacio físico. Por último, se solicitaba describir y ordenar, a partir de las percepciones individuales y el grado de importancia concebido, las barreras que atentan contra la inclusión de estudiantes con discapacidad en los centros de educación superior.

Entre los resultados obtenidos resalta que solo en dos universidades mostraban evidencias en relación a acciones de sustento al acceso pleno en tanto presentaron algunas adaptaciones curriculares. La generalidad, sin embargo, se caracterizó por un irrelevante empleo de recursos informáticos apoyados en la TICs para garantizar el pleno acceso a la información y, a pesar de que se muestran evidencias de conocer y dominar el marco normativo que regula las acciones del Sistema de Educación Superior en materia de integración de personas con discapacidad, las iniciativas existentes para integrarlas son escasas, aisladas y estructuralmente desintegradas y el diseño, implementación y sistematización de las ayudas técnicas personalizadas a estudiantes con discapacidad son limitados. Es de señalar que, al menos en el plano demostrativo (presentación documental, páginas web, etc.), ofrecen un tratamiento especial en el orden financiero a personas con discapacidad (becas, ayudas económicas y aranceles diferenciados). Sin embargo, las acciones del claustro continúan sustentándose en el esfuerzo individual de cada docente sin que se logre una actuación sistémica en tal sentido, por lo que persisten dificultades en la integración educativa de personas con discapacidad a nivel universitario donde los contenidos académicos, las alternativas didácticas y el nivel de especialización requieren de estrategias específicas y bien pensadas.

De esta manera, las principales barreras reconocidas por los participantes en el estudio y ordenas por ellos según el grado de importancia las relacionan, en primer lugar, con el profesorado (preparación del claustro docente para sumir la realidad inclusiva en el aula universitaria) a lo que sigue por orden de importancia las barreras preuniversitarias, relacionadas con el acceso, permanencia y progreso en las etapas educativas anteriores a la universidad. Los actores universitarios entrevistados coincidieron en que en tercer lugar, entre las barreras limitantes se encuentran las relacionadas con los medios didácticos y recursos de apoyo, es decir, con la implementación de tecnologías para la igualdad de oportunidades de todos en los escenarios educativos. A continuación, los entrevistados concuerdan en que otra de las barreras es la relacionada con la disponibilidad de la información para todos los estudiantes por igual. Por último, mencionan por igual las barreras relacionadas con los paradigmas educativos tradicionales, o lo que es lo mismo, las barreras derivadas de modelos tradicionales y poco flexibles de enseñanza y aprendizaje y aquellas que se relacionan directamente a la persistencia de actitudes sociales discriminatorias.

Ante esta panorámica se evidencia la necesidad de dotar al proceso de gestión universitaria y de profesionalización del claustro de un modelo (figura 1) que emplee como vía de implementación un Programa de Carrera Docente, cuyo objetivo fundamental se dirija a la profesionalización del docente universitario para el tratamiento a las personas con discapacidad de manera que 
permita al claustro de las IES pensar, reflexionar, opinar, emitir juicios, participar y buscar nuevas estrategias que propicien formar parte activa de este proceso. El modelo que se propone está encaminado no solo a fortalecer y profesionalizar al claustro, sino que pretende también preparar, en lo que se ha llamado Programa de Carrera Docente, a sus profesores y estudiantes de tercer nivel para que se conviertan en los futuros docentes universitarios que transformarán al Ecuador y garanticen la contextualización práctica del eslogan del pleno acceso y el tratamiento a la diversidad al interior de las IES. En este sentido, el Programa de Carrera Docente concibe tres momentos: el primero dedicado a la profesionalización pedagógica como un proceso continuo que atendiendo a las diferentes etapas organizadas facilitará adiestrar, formar y perfeccionar a los docentes en ejercicio, el segundo dirigido a la preparación de estudiantes de tercer nivel y docentes sin formación pedagógica en el dominio de los contenidos de la didáctica con el propósito de incidir en la transformación de la educación ecuatoriana, garantizando el relevo del claustro universitario existente en el país y el tercero que se ocupa de la formación científica del claustro universitario ecuatoriano.

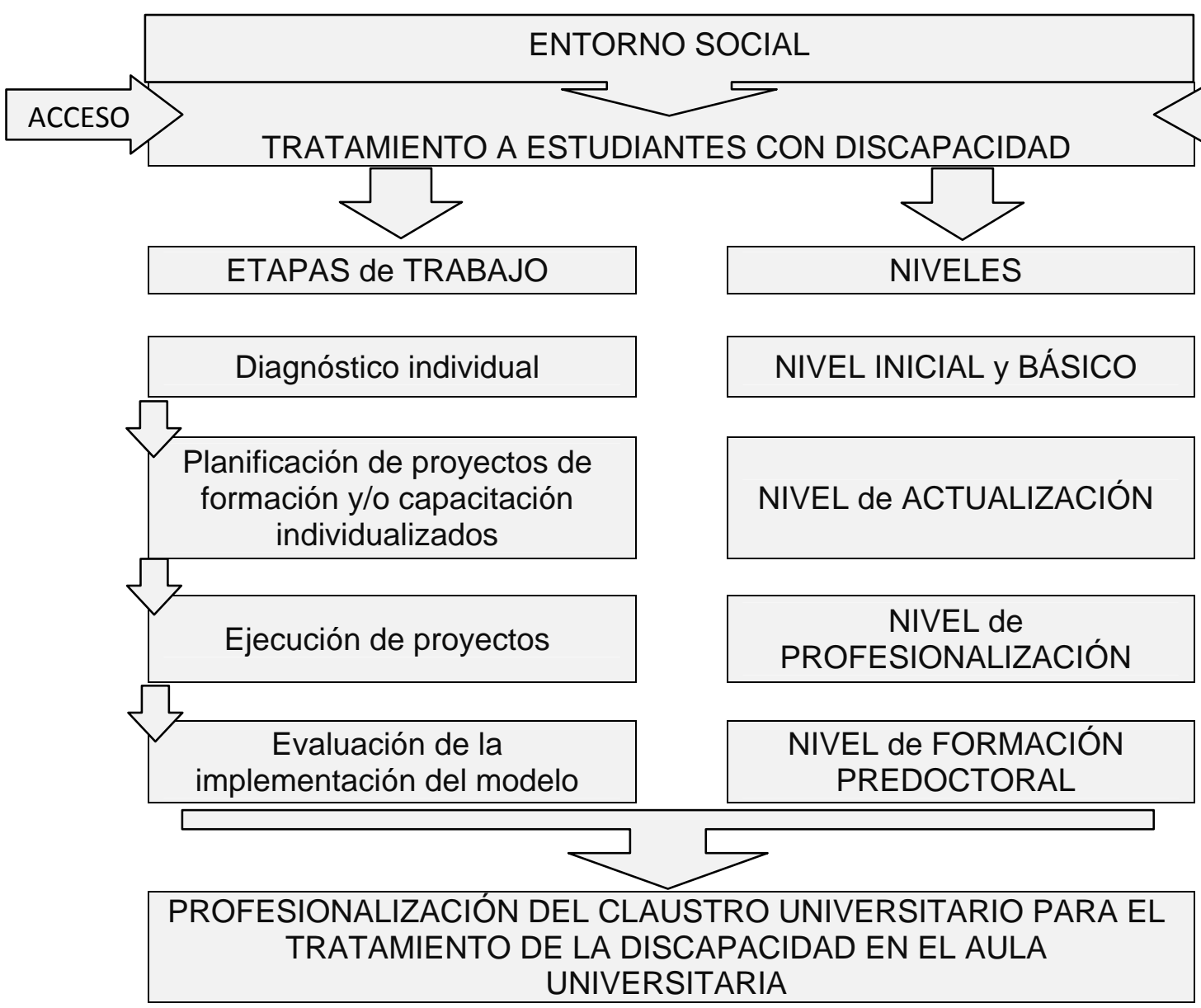

Fig. 1: Modelo para la gestión del acceso y la retención de estudiantes con discapacidad a las IES

El Programa de Carrera Docente del profesorado universitario tiene entre sus objetivos garantizar la continuidad del claustro universitario a partir de la preparación pedagógica paralela de estudiantes universitarios de tercer nivel, facilitar el proceso de iniciación del profesor que posee formación académica y científica sobre la disciplina que explica; pero no dispone de los fundamentos pedagógicos para dirigir el proceso de enseñanza aprendizaje en la educación superior, propiciar el adiestramiento docente y la formación pedagógica por niveles del claustro universitario activo en la educación superior ecuatoriana, dirigir la formación académico investigativa del claustro universitario encaminada al perfeccionamiento continuo y al desarrollo de competencias para investigar sobre la propia práctica docente o en su área de conocimiento y garantizar el adecuado tratamiento, desde lo pedagógico, lo sociológico, lo psicológico y lo tecnológico, a las personas con discapacidad que accedan a las instituciones de educación superior. 
La instrumentación del Modelo supone la conformación de un equipo cuya función principal se centra en la organización, coordinación del programa y medición de los impactos de los resultados del mismo así como la presencia de expertos en la profesionalización de docentes universitarios en Pedagogía, Didáctica General, Didácticas Específicas y Gestión Universitaria quienes impartirán los módulos temático, talleres y realizarán el acompañamiento necesario en el proceso de investigación, formarán equipos de investigación - acción para el estudio de los principales problemas existentes en el contexto educativo ecuatoriano y formularán propuestas y asesorías para la mitigación de las insuficiencias plasmadas en los diagnósticos realizados. Ellos serán protagonistas, junto al resto de la comunidad universitaria, del acompañamiento en la implementación del mismo.

\section{CONCLUSIONES}

La investigación realizada, en su proceso dialéctico, propicia una comprensión holística del objeto de estudio y permite arribar a las siguientes conclusiones:

A pesar de constituir una Política de Estado, las IES, salvo contadas excepciones, las IES ecuatorianas estudiadas no poseen registro de implementación de programas, sistemas de apoyo y sensibilización, adaptaciones curriculares, tecnológicas, informacionales y arquitectónicas, que faciliten el acceso de las personas con discapacidad a la universidad. Se mantiene la ausencia de programa o proyectos gubernamentales, liderados desde la Subsecretaría de Educación Superior del Ecuador, que regule y coordine esfuerzos en relación a la integración educativa superior de personas con discapacidad. Puede asegurarse que la inclusión del estudiantado con discapacidad en la educación superior es una tarea que se muestra aún inconclusa en las universidades ecuatorianas, y que los planteamientos hacia la sociedad inclusiva se reafirman como una demanda generalizada.

El modelo propuesto como resultado de esta investigación permite organizar, planificar, controlar y evaluar el proceso de profesionalización del claustro universitario y la preparación del estudiantado de tercer nivel para enfrentar el reto de garantizar la permanencia de estudiantes con discapacidad en las aulas universitarias. La propuesta, a criterio de los expertos que la evaluó, es novedosa, pertinente y científicamente fundamentada. Por todo lo anterior se puede aseverar que la dirección consciente, eficaz y eficiente del acceso y la retención de estudiantes con discapacidad a las carreras universitarias solo es posible cuando se expresa en el contexto de la relación función-proceso y, en tal sentido, se organiza su gestión.

\section{REFERENCIAS}

Alonso, A. y Díez, E. Universidad y discapacidad: indicadores de buenas prácticas y estándares de actuación para programas y servicios. SIGLOCERO Revista Española sobre Discapacidad Intelectual, Vol 39 (2), Núm. 226, 82 - 98 (2008).

Parreño, M. J. e I. de Araoz. El impacto de la Convención Internacional sobre los Derechos de las Personas con Discapacidad en la legislación educativa española. Madrid. Ediciones Cinca, (en línea), 2011.

http://www.convenciondiscapacidad.es/ColeccionONU_new/Impacto\%20de\%20la\%20C.6.pdf Acceso: mayo 07 (2012).

Aponte, E. Desigualdad, Inclusión y Equidad en la Educación Superior en América Latina y el Caribe: Tendencias y escenario alternativo en el horizonte 2021. En C. R. 2008, Tendencias de la Educación Superior en América Latina y el Caribe. Cartagena: CRES

Arnaiz, P. Educación inclusiva: Una escuela para todos. Málaga: Aljibe. (2003)

Baron, S., Phillips, R., Stalker, K. Barriers to training for disabled social work students, Disability and Society, 11, 361-377 (1996)

Brovetto, J. Formar para lo desconocido. Apuntes para la teoría y práctica de un modelo universitario en construcción. Serie "Documentos de Trabajo № 5. Universidad de la República. Montevideo - Uruguay (1994). 
Chiroleu, A. Políticas públicas de inclusión en la educación superior los casos de Argentina y Brasil. ProPosições vol.20 no.2 Campinas , pp. 141-166, May/Aug. 2009, (en línea) http://dx.doi.org/10.1590/S010373072009000200010) Acceso: mayo 08 (2012).

Chiroleu, A. (2011). La educación superior en américa latina: ¿problemas insolubles o recetas inadecuadas?. Avaliação (Campinas) , vol.16, n.3, pp. 631-653. (en línea), 2011. http://dx.doi.org/10.1590/S1414-40772011000300008) Acceso: abril 18 (2012).

Constitución de la República del Ecuador (en línea), 2008, http://www.asambleanacional.gov.ec Acceso: octubre 20, (2010).

Doyle, C.; Robson, K. Accessible Curricula: good practice for all Cardiff: University of Wales, Institute Cardiff Press (en línea), 2002. http://www.techdis.ac.uk/pdf/curricula.pdf Acceso: febrero 21 (2012)

Echeita, G. El proceso de inclusión educativa en España. ¡Quien bien te quiere te hará Ilorar!, CEE. Participación Educativa, 18, noviembre 2011, 117-128, (en línea), 2011. http://www.educacion.gob.es/revistacee/pdf/n18-echeita-sarrionandia.pdf Acceso: enero 11 (2012)

Fox, D. J. El Proceso de investigación en educación, 23-58, Pamplona: EUNSA (1981)

Freewood, M. y Spriggs, L. Striving for genuine inclusion: academic assessment and disabled students, in Rust, C. (ed.) Improving Student Learning Theory and Practice - 10 years on. Oxford: The Oxford Centre for Staff and Learning Development, Oxford Brookes University, 353-362 (2003)

Fuller, M., Healey, M., Bradley, A. y Hall, T. Barriers to learning: a systematic study of the experience of disabled students in one university, Studies in Higher Education 29 (3), 303-318 (2004a)

Fuller, M., Bradley, A. y Healey, M. Incorporating disabled students within an inclusive higher education environment, Disability and Society 19, 455-468 (2004b)

García, C. Una escuela común para niños diferentes. La integración escolar, 2-51, Barcelona: EUB (1993).

García, C. Educación y diversidad. Málaga: Aljibe, 23-42 (2005)

Gravestock, P.; Healey, M. Providing Learning Support for Disabled Students undertaking Fieldwork and Related Activities: A set of six guides. Cheltenham: Geography Discipline Network, University of Gloucestershire, 13-30 (2001).

Informe de la Misión Manuela Espejo, (en línea), 2010.

http://www.vicepresidencia.gob.ec/programas/manuelaespejo/mision Acceso: noviembre 15 (2010)

Jiménez, P. y Vilá, M. De la educación especial a la educación en la diversidad. Málaga: Aljibe, 1-32 (1999).

Kovadloff, S. La educación y la construcción de ciudadanía. En: Jaim Etcheverry, G. y col. Op. cit. (2003)

Maingon, R,. Caracterización de los estudiantes con discapacidad: Caso: Universidad Central de Venezuela. Rev. Ped. abr. 2007, vol.28, no.81, 43-79, (en línea), 2007.

http://www.scielo.org.ve/scielo.php?script=sci_arttext\&pid=S0798-97922007000100003\&lng=es\&nrm=iso Acceso: Mayo 7 (2012).

Muñoz, E. y Maruny, L. Respuestas escolares. Cuadernos de Pedagogía, 212, 11-14 (1993)

LOES (Ley Orgánica de Educación Superior), (en línea), 2010.

http://www.senescyt.gob.ec/c/document_library/get_file?uuid=29ad2ecf-15aa-4110-9d82-

e5994ca36d72\&groupld=10156 Acceso: octubre $2 \overline{1}$, (2010).

ONU, From Exclusion to Equality: Realizing the rights of persons with disabilities, Handbook for Parliamentarians on the Convention on the Rights of Persons with Disabilities and its Optional Protocol, Geneva 2007. Department of Economic and Social Affairs (UN-DESA), the Office of the United Nations high Commissioner for human Rights and the Inter-Parliamentary Union (en línea), 2007. http://www.unclef.com/disabilities/documents/toolaction/ipuhb.pdf Acceso: 11 abril 2011]. 
Organización de Estados Iberoamericanos para la Educación, la Ciencia y la Cultura (OEI). Metas educativas 2021. La educación que queremos para la generación de los Bicentenarios. Documento para debate, primera versión. Conferencia Iberoamericana de Ministros de Educación. Madrid: OEI (en línea), 2008. http://www.oei.es/metas2021/index.html Acceso: Marzo 10, (2011)

Paul, S. Students with disabilities in higher education: a review of the literature, College Student Journal, 34, 200-210 (2000).

Puigdellívol, I., La Educación Especial en la Escuela Integrada. Una perspectiva desde la diversidad. (7ma. ed.). Barcelona: Editorial GRAÓ, de IRIF, S.L. (2007).

Rodríguez, N. Integración de las personas con discapacidad en la Educación Superior en Ecuador. Digital Observatory for Higher Education in Latin America and the Caribbean (en línea), 2004. http://unesdoc.unesco.org/images/0014/001404/140488s.pdf Acceso: marzo 12 (2011).

Rose, D., Meyer, A.,. Universal Design for Learning. Journal of Special Education Technology, 15, pp. 67-70 (2000).

Sánchez, A y Torres González, J. A. Educación Especial. Una perspectiva curricular, organizativa y profesional. Madrid: Pirámide, 6-34 (2004).

Sánchez, A. y Pulido Moyano, R. El centro educativo: una organización de y para la diversidad. Granada. Grupo Editorial Universitario, 6-11 (2007).

Sánchez, A. La Universidad de Almería ante la integración educativa y social de los estudiantes con discapacidad. Ideas y actitudes del personal docente e investigador, Revista de Educación, № 354, 321322 (2011)

Tunnermann, C. La Educación Superior en el Umbral del Siglo XXI. UNESCO. Colección Respuestas, (1996)

Teachability (2000) Creating an Accessible Curriculum for Students with Disabilities. Glasgow: University of Strathclyde (en línea) http://www.teachability.strath.ac.uk/ Acceso: junio 1, (2010)

UNESCO Marco de Acción sobre las Necesidades Educativas Especiales (en línea), 1994. http://www.unesco.org Acceso: junio 2, (2010).

UNESCO. La Educación encierra un tesoro. Jacques Delors, (en línea) http://unesdoc.unesco.org/images/0010/001095/109590so.pdf Acceso: enero 23 (2011).

UNESCO. Declaración Mundial sobre la Educación Superior en el Siglo XXI: Visión y Acción (en línea), 1998. http://www.unesco.org/education/educprog/wche/declaration_spa.htm Acceso: junio 2, (2010).

UNESCO, Overcoming Exclusion through Inclusive Approaches in Education: A Challenge and a Vision, Conceptual Paper, Paris, (en línea), 2003. http://unesdoc.unesco.org/ima Acceso: junio 2 (2011).

UNESCO Superar la exclusión mediante planteamientos integrales en la educación. Un desafío \& una visión (en línea), 2003. http://www.unesco.org Acceso: junio 2, (2010).

UNESCO/IESALC, Informe Sobre la Educación Superior en América Latina y el Caribe 2000-2005. La Metamorfosis de la Educación Superior. Caracas: Instituto Internacional de la UNESCO para la Educación Superior (en línea), 2006. http://www.iesalc.unesco.org.ve Acceso: enero 23 (2011).

UNESCO-IESALC. Educación Superior, Colaboración Intercultural y Desarrollo Sostenible/Buen Vivir. Experiencias en América Latina / coordinado por Daniel Mato.- Caracas (en línea), 2009. http://www.iesalc.unesco.org.ve Acceso: enero 23 (2011). 